\begin{abstract}
A major challenge in biophotonics is multimodal imaging to obtain both morphological and molecular information at depth. We demonstrate a hybrid approach integrating optical coherence tomography (OCT) with wavelength modulated spatially offset Raman spectroscopy (WM-SORS). With depth colocalization obtained from the OCT, we can penetrate $1.2 \mathrm{~mm}$ deep into the strong scattering media (lard) to acquire up to a 14-fold enhancement of a Raman signal from a hidden target (Polystyrene) with a spatial offset.

Our approach is capable of detecting both Raman and OCT signals for pharmaceutical particles embedded in turbid media and revealing the white matter at depth within a $0.6 \mathrm{~mm}$ thick brain tissue layer. This depth resolved label-free multimodal approach is a powerful route to analyze complex biomedical samples.
\end{abstract}

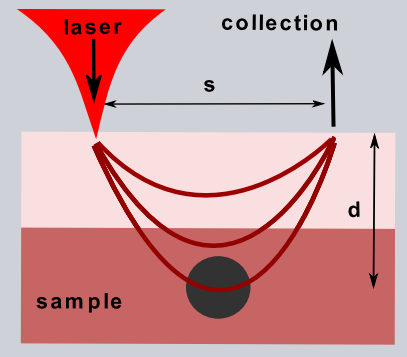

(a) Sample plane

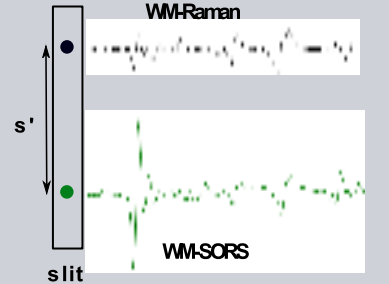

(b) Spectra acquisition

\title{
Depth-resolved multimodal imaging: Wavelength Modulated Spatially Offset Raman Spectroscopy with Optical Coherence Tomography
}

\author{
Mingzhou Chen ${ }^{1,{ }^{*}}$, Josep Mas ${ }^{1}$, Lindsey H. Forbes ${ }^{2}$, Melissa R. Andrews ${ }^{2,}{ }^{3}$, and Kishan \\ Dholakia $^{1, *}$
}

\section{Introduction}

Recently there has been increasing interest for the development of multimodal imaging [1] in biophotonics. Spectroscopy may reveal molecular markers of disease with high specificity, while imaging can unravel the microstructures of tissue with high sensitivity. Thus an approach which can simultaneously make use of both approaches, for example the integration of combination of optical coherence tomography (OCT) and Raman spectroscopy [2,3], would yield simultaneous morphological and molecular information and ultimately lead to an enhancement of sensitivity and specificity for identification that is not possible with either modality on its own [4-10].

Raman spectroscopy has been widely used for pharmaceutical detection $[11,12]$ and disease diagnosis [7, 13-15]. Whilst OCT can penetrate several millimeters deep into the samples while scanning over a large transverse area (several $\mathrm{mm}^{2}$ ), Raman signals from the deeper layers are very weak as the photons experience stronger diffusion along the path to and from the deeper areas. Thus conventional Raman approach means information beyond 100um is difficult. To address this spatially offset Raman spectroscopy (SORS) [16] has been applied to increase the Raman penetration depth into samples by spatially moving the collection point away from the excitation point. The penetration depth is related to this offset and the optical properties of the sample [17]. In the most recent SORS approaches, this offset may be fixed or optimized by moving the collection optics/fibre manually $[18,19]$ or manipulated by a digital micromirror device (DMD) [20], though this adds a degree of complexity. It is to be noted that to date SORS (as with standard Raman acquisition) may be hampered by background fluorescence and this has not been addressed. Furthermore SORS has typically recorded point signals. Most crucially for this present work, SORS has not been employed for imaging nor any multimodal imaging application.

Our letter describes an integration of SORS with OCT with a number of key innovative attributes. For the first time we are able to obtain both OCT and Raman images at large depths ( $\mathrm{mm}$ ) and co-register data from images acquired by both modalities. Further, our implementation of SORS is in itself original, using wavelength modulation, so we can acquire fluorescence-free Raman spectra at different spatial offsets simultaneously using one single CCD camera. Finally, due to the geometry we use this is achieved in the absence of any moving parts. We term this original combination of wavelength modulated Raman spectroscopy (WMRS) $[21,22]$ and the technique of SORS, as WM-SORS. We emphasize that our system also uses the very same optics for the Raman and OCT paths which allows for co-registration of image data. This original ap-

${ }^{1}$ SUPA, School of Physics and Astronomy, University of St. Andrews, KY16 9SS, UK. ${ }^{2}$ School of Medicine, University of St. Andrews, KY16 9 TF, UK. ${ }^{3}$ Biological Sciences, University of Southampton, SO17 1BJ, UK (current address).

* Corresponding author: e-mail: mingzhou.chen@st-andrews.ac.uk or kd1@st-andrews.ac.uk 
proach to multimodal imaging will open up a new route for simultaneously obtaining molecular and morphological information from deep layers of biological samples whilst simultaneously removing any interfering fluorescence.

Our experimental results from complex lard-polystyrene phantoms show that our multimodal WM-SORS/OCT system can penetrate $1.2 \mathrm{~mm}$ deep into highly scattering media to acquire Raman signals from the hidden targets. With a spatial offset of $0.43 \mathrm{~mm}$, the system can achieve an enhancement factor as high as 14 . A range of experimental results with pharmaceutical samples and layered rat brain tissue samples show the ability of our system to reveal the hidden targets at depth $(>0.5 \mathrm{~mm})$ using both morphological and molecular information. Thus, Therefore, our approach opens up new prospects for analyzing complicated biological samples and simultaneously obtaining morphological and molecular information from extended 3D volumes in tandem for the first time.

\section{Materials and Methods}

\subsection{Wavelength modulated spatially offset Raman spectroscopy (WM-SORS)}

In SORS, the collected Raman photons can reliably recover biological Raman spectra from deep layers or hidden subjects due to a small offset, $s$, between the collection point and the excitation laser as shown in Fig. 1(a). Usually a movable collection fibre or a fibre bundle is needed to achieve different spatial offsets $s$ in order to obtain Raman signals from the target at different depths $d$. Without using any extra complicated optical component or optical fibre, our WMSORS setup is exactly the same as a standard free space Raman spectroscope. The collection spatial offset is actually fulfilled by the two dimensions of the imaging CCD camera on the spectrometer. As shown in Fig. 1(b), the laser spot and the collection point are imaged on the slit of the spectrometer which is then relayed onto the CCD camera in order to get the spectral information. Therefore, the rows of binned pixels on CCD contain SORS information when they are not exactly on the rows of pixels which correspond to the image of laser spot. The spatial offset $s^{\prime}$ on the CCD plane can be converted into the physical spatial offset $s$ on the sample plane with knowledge of the magnification factor of 2.78 in the optical system. In this way, one can easily take standard Raman spectra and SOR spectra, at different offsets simultaneously, by extracting the spectra from different rows of CCD pixels in the spectrometer. From our system, one standard Raman spectrum and eight SOR spectra at different offsets can be extracted from each CCD frame. In order to get WMR/WMR-SOR spectra, five frames from the CCD camera of spectrometer need to be acquired when the laser is tuning its wavelength over $1 \mathrm{~nm}$ with a step of $0.2 \mathrm{~nm}$. Then using the wavelength tuning step of $0.2 \mathrm{~nm}$ as a parameter, one can calculate one WMR spectrum from five standard Raman spectra and eight WM-SOR spectra from each five SOR spectra at each offset [22]. Similar to the method used in our previous work [21,22], each WM-SOR spectrum is calculated from five standard Raman spectra with the step of wavelength as a parameter using principal component analysis (PCA). In this way, both WMR spectra and WM-SOR spectra can be obtained simultaneously from the frames on the same CCD camera in one single shot using the analyzing method described previously. With the benefit of background fluorescence suppression using WMRS [21], the obtained WM-SOR signal has an enhancement of $20 \%$ to $30 \%$ higher than standard SORS from one of our detailed study (data is not shown here). Therefore, we will focus on the WM-SORS for the rest of this letter.

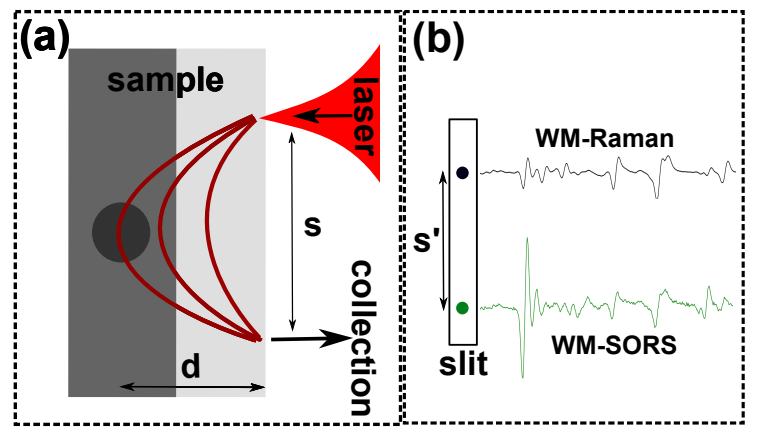

Figure 1 Schematic of WM-SORS. (a) shows the laser, sample and spatially offset collection point. (b) shows the images of laser point and spatially offset collection point and their corresponding WM Raman spectra when the grating is in use.

\subsection{Hybrid WM-SORS and OCT system setup}

In order to obtain both morphological and molecular imaging at depth, we integrate OCT and WM-SORS into a hybrid system as shown in Fig. 2. In this setup, a low NA objective (Thorlabs, LSM02-BB) is used for both OCT and WMSORS. A tunable $785 \mathrm{~nm}$ laser source (Spectra-Physics, $1 \mathrm{~W}$, Ti:Sapphire 3900s) is used as an excitation laser source for WM-SORS with a wavelength tuning range of $1 \mathrm{~nm}$. The laser power at the sample plane is about $100 \mathrm{~mW}$. A broadband SLD (Superlum, S850, central wavelength $850 \mathrm{~nm}, \Delta \lambda$ $=30 \mathrm{~nm}$ ) is used as an OCT light source. To avoid any signal loss, a flip mirror $\left(\mathrm{F}_{1}\right)$ is used to switch between OCT mode and WM-SORS mode. As the optical refractive index of the sample is unknown, it needs to calibrate the relationship between the spatial offset $s$ and the depth information $d$ for different samples. With such a hybrid system, WM-SOR spectra or Raman images from hidden objects deep in the sample can be acquired with the depth information $d$ coregistered using OCT images. In this way, we can avoid the complicated calibration procedure of $s$ and $d$ in our system. Note that a low NA objective is used for both OCT and WM-SORS which may reduce the performance of Raman spectroscopy. 


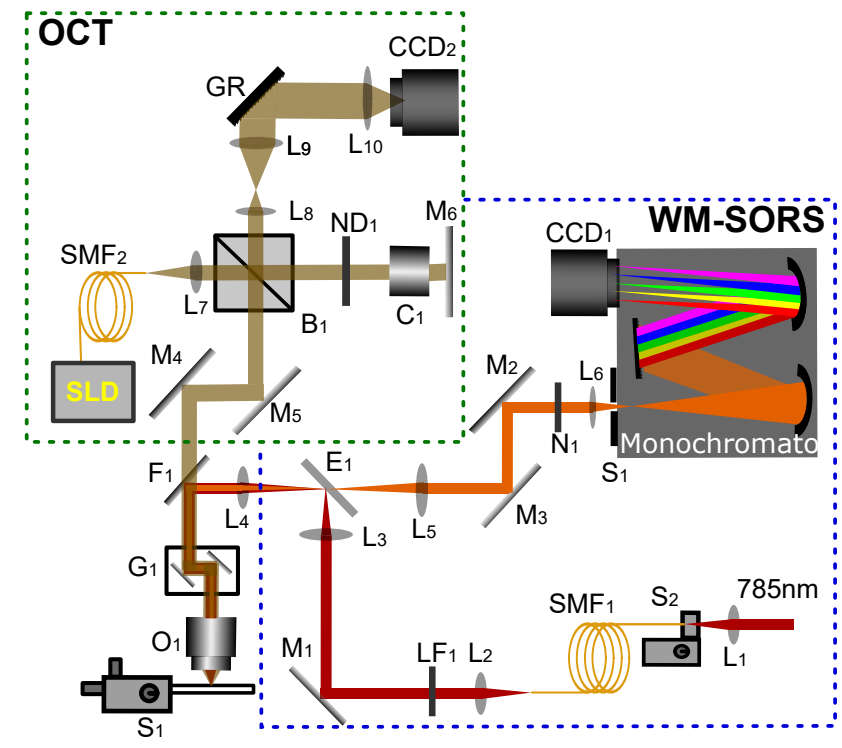

Figure 2 Schematic of hybrid WM-SORS and OCT system. $L_{1}$ - $L_{10}$ : lenses. $M_{1}-M_{6}$ : mirrors. $F_{1}$ : a flipping mirror for switching between OCT and WM-SORS. GR: a transmission grating (1200 lines/mm, coated for 700-960nm) (drawn in a reflection mode). $\mathrm{G}_{1}$ : a 2D Galvo-mirror (Thorlabs, GVS002). $\mathrm{O}_{1}$ : an OCT objective (Thorlabs, LSM02-BB) and $\mathrm{C}_{1}$ : an optical path matching glass cylinder (Thorlabs, LSM02DC). SLD: a broadband OCT light source (Superlum, S850). SMF 1 and $\mathrm{SMF}_{2}$ : single mode fibres. A spectrometer with a cooled CCD camera $\left(\mathrm{CCD}_{1}\right.$, Anor Technology, Newton, 1024x256 pixels) and a Monochromator (Andor SR303i) is used to acquire Raman spectra. $C_{C D}$ : a line CCD (e2v AViiVA $E_{1}, 2048$ pixels). $E_{1}$ : an edge filter (Semrock, LPD01-785RU-25). $\mathrm{N}_{1}$ : a notch filter (Semrock, NF03-785E-25). LF 1 : a laser line filter (Semrock, LD01-785). $\mathrm{S}_{1}$ : a single slit (100um in width). $\mathrm{ND}_{1}$ : neutral density filter. $B_{1}$ : a 50:50 beam splitter. Green dashed lines and blue dashed lines enclose the OCT section and the WM-SORS section of the setup respectively.

\subsection{Polystyrene/lard phantom}

A piece of polystyrene sheet ( $1.5 \mathrm{~mm}$ thickness) is diagonally inserted into a lard block as shown by the OCT image in Fig. 3. The Raman laser is focused on the lard surface and both standard Raman spectra and WM-SOR spectra are taken using an integration time of 10s. Same integration time of $10 \mathrm{~s}$ is used for the pharmaceutical particles/lard phantom and the rat brain tissue. All depth information in this paper represents optical depth registered with OCT images. This polystyrene/lard phantom is then used to characterize our WM-SORS/OCT system.

\subsection{Pharmaceutical particles/lard phantom}

The phantom is made by sandwiching granulated aspirin tablets and ibuprofen tablets between two slices of lard (about $1 \mathrm{~mm}$ in thickness). Raman spectra were acquired from standard pure lard, aspirin and ibuprofen tablets with the same system using an integration tie of 2s. From the Raman spectra of lard, aspirin and ibuprofen as shown in

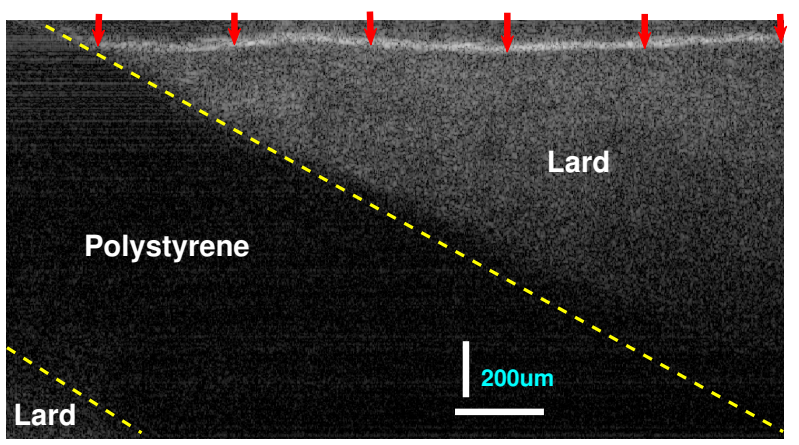

Figure 3 OCT image shows the interface of lard and a polystyrene sheet. Two yellow dash lines show the interface of polystyrene sheet and lard. Raman spectra were acquired at the positions on lard surface as indicated by the red arrows. The corresponding WM-SOR/SOR spectra were acquired at the positions in the perpendicular plane while having a spatial offset $s$ away from the red arrows. Scale bars indicate $200 \mu \mathrm{m}$ in horizontal and vertical directions respectively.

Fig. 4, one can find several characteristic Raman peaks, for example lines at $840 \mathrm{~cm}^{-1}, 1188 \mathrm{~cm}^{-1}, 1430 \mathrm{~cm}^{-1}$ and $1595 \mathrm{~cm}^{-1}$, which can be used for identifying these three compounds clearly.

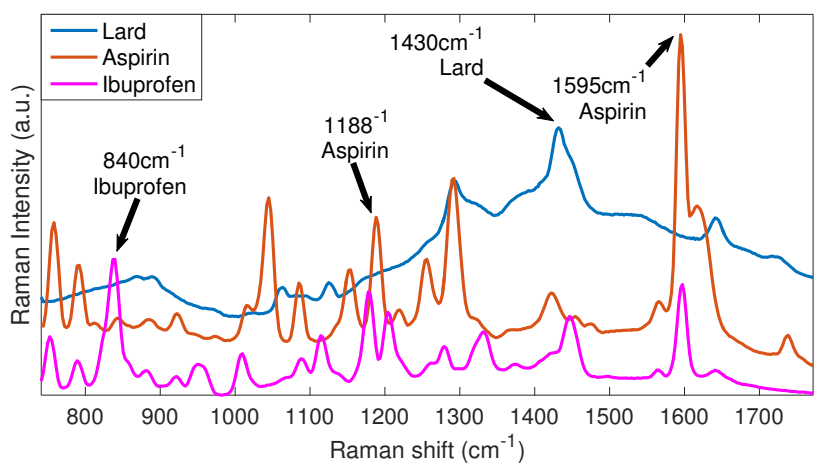

Figure 4 Standard Raman spectra from pure aspirin tablet, ibuprofen tablet and lard with same integration time of $2 \mathrm{~s}$.

\subsection{Rat brain tissue}

Experiments were conducted in accordance with the UK Animals (Scientific Procedure) Act, 1986. Food and water were provided ad libitum and there was 12 hour light/dark exposure. Brain tissue obtained from adult Sprague Dawley rats was fixed by transcardial perfusion with phosphatebuffered saline (PBS) followed by $4 \%$ paraformaldehyde (PFA) and postfixation in 4\% PFA. Cryoprotection was performed overnight in $20 \%$ sucrose in $0.1 \mathrm{M}$ PBS. Brain tissue was sectioned in the coronal plane on a sliding microtome (Leica) at a thickness of $0.1,0.2$ and $0.4 \mathrm{~mm}$. Sections were stored in PBS until used for imaging.

A rat brain tissue sample is formed by one layer of fixed rat brain tissue with a thickness of $0.2 \mathrm{~mm}$ being aligned on 
top of another layer with a thickness of $0.4 \mathrm{~mm}$. The white matter within one tissue layer is not directly apposed to the white matter within the other tissue layer. The sample was mounted on a thick quartz slide in a petri dish filled with PBS solution in order to keep the sample moist during the experiments.

\section{Results}

\subsection{System characterization with polystyrene/lard phantom}

Due to the distance from the laser focus and the high scattering property of lard, the non-offset WM Raman peak intensities from polystyrene sheet $\left(1001.4 \mathrm{~cm}^{-1}\right)$ dramatically decrease when the polystyrene sheet is buried deeper into the lard, as shown in Fig. 5.

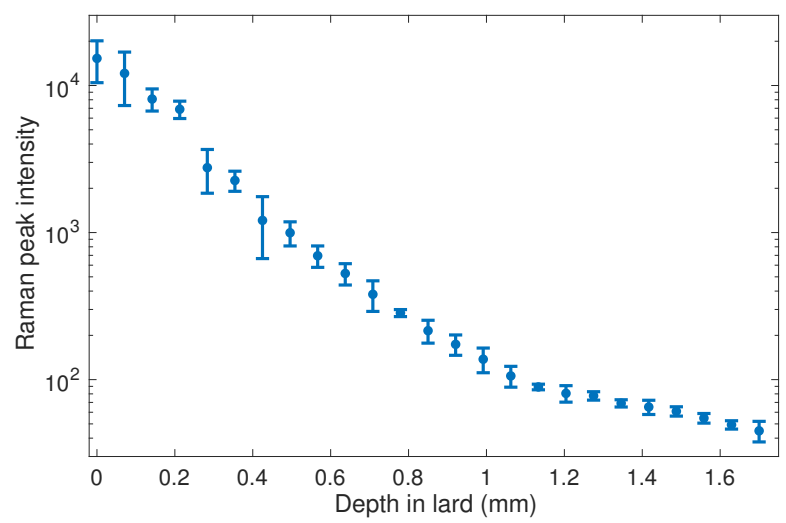

Figure 5 Non-offset WM Raman intensity of a polystyrene peak $\left(1001.4 \mathrm{~cm}^{-1}\right)$ as a function of the depth of the polystyrene in lard (in logarithmic scale).

Figure 6 shows a non-offset WM Raman spectrum $(s=$ $0 \mu \mathrm{m}$ ) and WM-SOR spectra with different spatial offsets $s$ which are acquired simultaneously when the polystyrene sheet is at $0.6 \mathrm{~mm}$ (registered by the OCT image) deep in the lard. All these spectra were normalized by the lard peak $\left(1430 \mathrm{~cm}^{-1}\right)$. The normalized Raman peak intensity of polystyrene at $1001.4 \mathrm{~cm}^{-1}$ reaches a maximum when $s$ is $170 \mu \mathrm{m}$ and it decreases as $s$ increases further. These results show the effectiveness of WM-SORS for recovering signals from the hidden samples in the deeper layers, even for the samples in the layers with high light scattering properties such as lard.

Figure 7 shows the dependence of the relative intensities of the polystyrene Raman peak $\left(1001.4 \mathrm{~cm}^{-1}\right)$ on the spatial offset $s$ and the thickness of lard layer $d$ which is co-registered by OCT image as shown in Fig. 3. The laser is always focused on the surface of the sample. The depth information $d$ indicate the location of the polystyrene in the lard layer at the position of laser focus. As the spatial offset increases, the Raman intensity ratio from the polystyrene

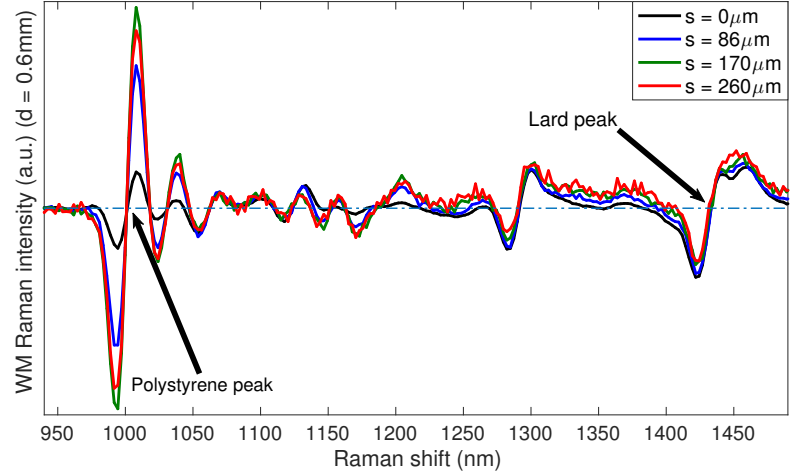

Figure 6 WM Raman spectra taken at different spatial offsets and normalized by the lard peak $\left(1430 \mathrm{~cm}^{-1}\right)$ from the phantom shown in Fig. 3. The optical depth of lard is $d=0.6 \mathrm{~mm}$ measured by OCT images. The dash-dotted line shows the zero-crossing of WM Raman spectra. Traditional Raman peaks are represented by the zero-crossings in WM Raman spectra while their peak intensity corresponds to the peak-valley value in WM Raman spectra.

with a thicker layer of lard increased more rapidly, indicating that the thicker the top layer, the more enhancement the Raman signal from hidden polystyrene can be achieved. According to each ratio curve with the specific value lard thickness $d$, there is obviously an optimized spatial offset for each lard thickness $d$ to record the highest enhancement for the WM-SORS signals.

\subsection{Detecting pharmaceutical particles in Lard with WM-SORS/OCT}

In this section, our WM-SORS/OCT system is used to take both morphological and molecular images from aspirin and ibuprofen particles buried deep in a lard block. Figure 8 $(\mathrm{a}-\mathrm{d})$ shows the OCT slice images at different depth. One can clearly see there are two hidden objects at a depth of about $1 \mathrm{~mm}$ in the lard. However, it is difficult to discriminate which pharmaceutical particle we are observing. WMR spectra are acquired from the same sample, which are shown in Fig. $8(e-p)$. Each $42 \times 42$-pixel Raman image using one characteristic Raman peak intensity is corresponding to the same area as in OCT images. Different spatial offsets $s$ are used in order to obtain the depth information from WM-SORS images. For the non-offset WM Raman with $s=0 \mu m$, the aspirin particle in the deep lard can be seen as it is quite big and shallow in the lard ( $8(\mathrm{e})$ ). However, the smaller ibuprofen particle which is deeper in the lard can not be detected at all ( 8 (i)). One can see the strong lard signals present as background noise in all non-offset WM Raman images (Fig. 8 (e, i, m)). From WM-SORS images (Fig. 8 (f-h, j-l)), one can see the Raman signals from those pharmaceutical particles and clearly one can also identify the aspirin particle and the ibuprofen particle. In the WMSORS images from lard peaks (Fig. 8 (n-p)), the dark area indicates there is no lard where the aspirin particle lies. 


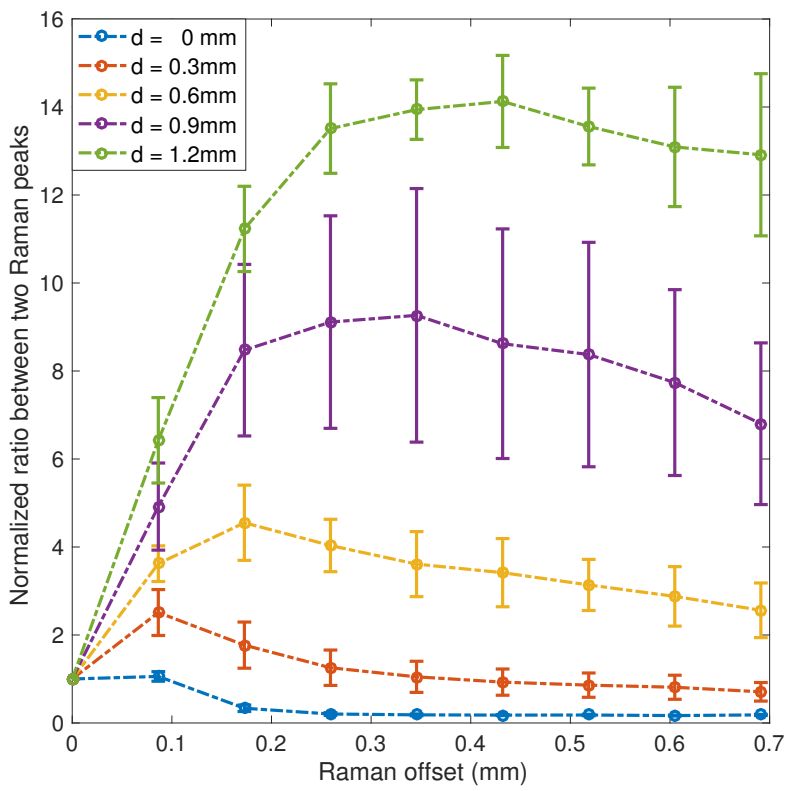

Figure 7 Ratios between two Raman peaks (polystyrene/lard) as a function of spatial offset $s$. Each colored curve corresponds to a different lard thickness $d$, that is co-registered with the OCT image. Two peaks are chosen at the largest polystyrene peak at $1001.4 \mathrm{~cm}^{-1}$ and the largest lard peak at $1430 \mathrm{~cm}^{-1}$. All ratios are normalized by the first ratio when no offset is used. Standard deviations were obtained from six measurements on the samples for each condition.

\subsection{WM-SORS/OCT imaging on layered rat brain tissue}

To further demonstrate the potential of the WM-SORS/OCT system, a layered rat brain tissue sample, as shown in the insets (a) and (b) in Fig. 9, is used for acquiring both OCT and Raman images. Using the OCT system, we can acquire an OCT B-scan image as shown in Fig. 9. The tissue-air interface is quite clear due to the change in refractive index while the tissue-tissue interface is very blurred. The white matter within both layers can be seen and are indicated by the green double ended arrows. The Raman laser also scans along the OCT scanning line to acquire 100 non-offset WMR spectra and 100 WM-SOR spectra simultaneously. The Raman images and OCT images show the same slice of the sample due to the co-alignment between both optical axis in WM-SORS and OCT. As clearly shown by the brown and cyan curves plotted on top of the OCT image in Fig. 9, the Raman signals (Raman peak intensities at $1430 \mathrm{~cm}^{-1}$ ) from the white matter in the bottom tissue layer are enhanced by a factor of two when a spatial offset $\mathrm{s}=86 \mu \mathrm{m}$ is used.

Then, we use the same sample and do an OCT C-scan over an area of $2.5 \times 2.5 \mathrm{~mm}$. OCT slice images at different depths are shown in Fig. 10 (a-d). In these OCT images, one can clearly distinguish the white matter in the top layer $(a, b)$ from the white matter in the bottom layer $(c, d)$. Some artifacts which may be caused by the liquid between two layers can also be detected and are seen in Fig. 10 (c, d).
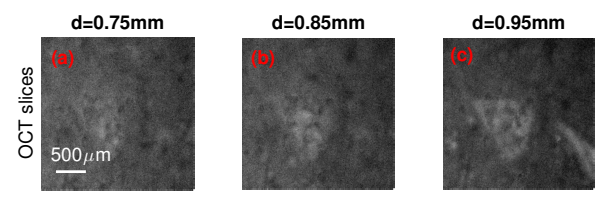

$d=1.05 \mathrm{~mm}$
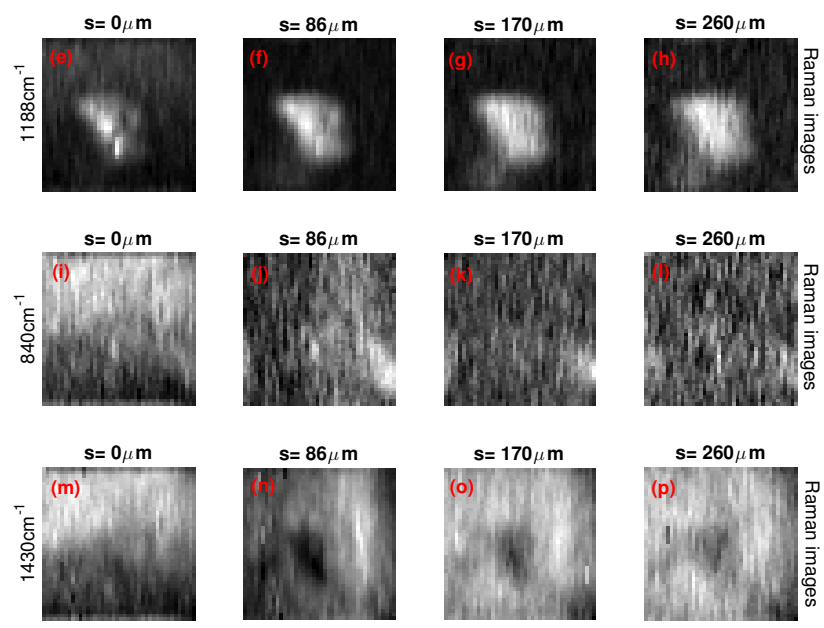

Figure 8 OCT and WM-SORS images for a phantom of aspirin and ibuprofen sandwiched by lard layers. (a)-(d) show the OCT slices at depths of $0.75 \mathrm{~mm}, 0.85 \mathrm{~mm}, 0.95 \mathrm{~mm}$ and $1.05 \mathrm{~mm}$ respectively. Note that the OCT images are averaged over 10 slices around the depth in order to increase the contrast. (e)-(h) show Raman images at $1188 \mathrm{~cm}^{-1}$ of aspirin with the offset of 0,86 , 170 and $260 \mu \mathrm{m}$ respectively. (i)-(I) show Raman images at 840 $\mathrm{cm}^{-1}$ of ibuprofen with the offset of $0,86,170$ and $260 \mu \mathrm{m}$, respectively. (m)-(p) show Raman images at $1430 \mathrm{~cm}^{-1}$ of lard at the offset of $0,86,170$ and $260 \mu \mathrm{m}$, respectively. Note that all Raman images are smoothed with a $3 \times 3$ median filter to increase the contrast.

Raman images using different spatial offsets are acquired on the same area based on the $1430 \mathrm{~cm}^{-1}$ peak intensities. With zero spatial offset as shown in Fig. 10 (e), one can distinguish the white matter in the top layer but can not see clearly the white matter in the low layer. From WM-SORS images as shown in Fig. 10 (f-h), one can see the white matter in the bottom layer more clearly than the one in the top layer as the image contrast is substantially enhanced. Furthermore, the artifacts in the OCT images, as shown in Fig. 10 (c,d), do not exist in these WM-SORS images due to the specific molecular information acquired.

\section{Discussion and conclusion}

We have demonstrated a hybrid WM-SORS/OCT system which can perform multimodal imaging and acquire both morphological and molecular images in deep tissue or through turbid media. As with an established SORS system, our novel WM-SORS/OCT system retains the ability of acquiring both WMR spectra and WM-SOR spectra at different spatial offsets simultaneously on one single CCD camera without using any movable optics and addi- 


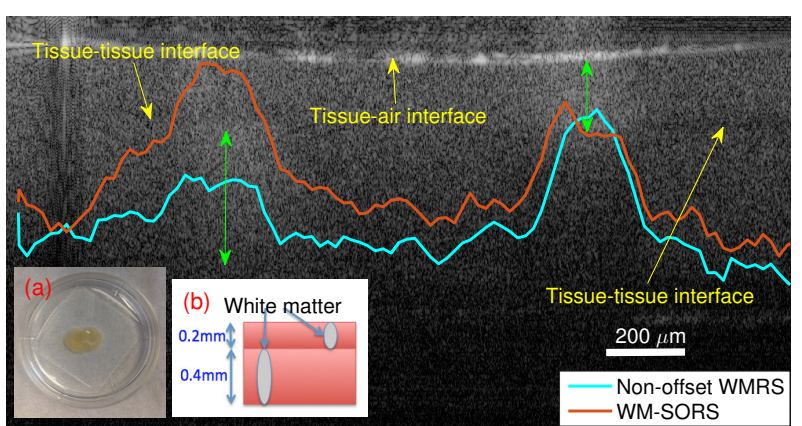

Figure 9 OCT B-scan image with Raman peak $\left(1430 \mathrm{~cm}^{-1}\right)$ intensities on fixed rat brain tissue. Cyan and brown curves show the Raman peak intensities at $1430 \mathrm{~cm}^{-1}$, across the same scanning line of OCT, acquired with non-offset WMRS and WM-SORS $(s=86 \mu \mathrm{m})$, respectively. Green double arrows show the position and depth of the white matter in two brain tissue layers. Tissue-air and tissue-tissue interfaces are indicated by the yellow arrows. Scale bar equals $200 \mu \mathrm{m}$ in this image. Inset (a) shows a photo of the brain tissue on a quartz slide in a petri dish and inset (b) shows a diagram of the two layers of brain tissue with white matter.
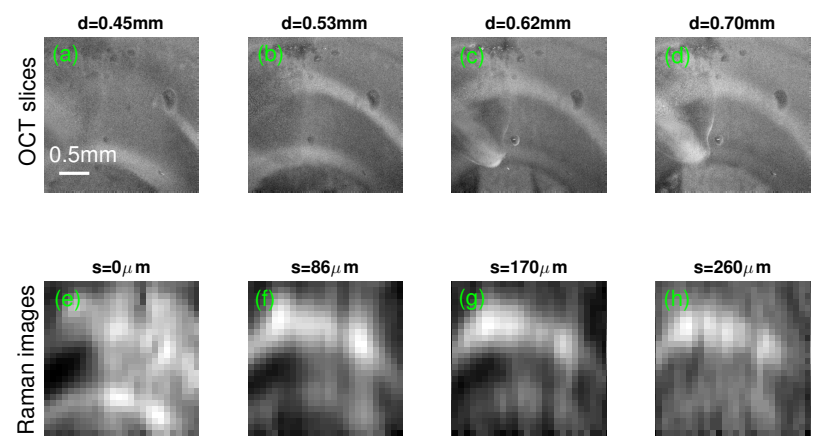

Figure 10 OCT and WM-SORS on brain tissue. (a) - (d) show OCT images of brain tissue at different depth. Note that OCT images are averaged over 10 slices around the depth in order to increase the contrast. (e) - (h) show Raman images (at $1430 \mathrm{~cm}^{-1}$ ) of the same brain tissue at different offsets. Note that Raman images are smoothed with a $3 \times 3$ median filter to increase the contrast.

tionally removes fluorescence. With the co-alignment of the OCT and the Raman probe beams, our system can penetrate $1.2 \mathrm{~mm}$ deep into strong scattering media (lard) to acquire Raman signals guided by OCT images. Using an optimized spatial offset in the lard/polystyrene phantom, the system can achieve a high enhancement factor of 14 . With the added benefit of removing any background fluorescence using WM-SORS, the Raman signal enhancement was $20 \%$ to $30 \%$ higher than standard SORS from one of our detailed study (data is not shown here). Using our approach, we have also successfully acquired Raman images from the white matter through a highly scattering brain tissue layer. The co-registered OCT and Raman images can reveal both structural and biochemical information from deep tissues which opens a new approach to analyze complicated biological and clinical samples. This hybrid approach has the potential to improve upon existing OCT methodologies for screening and diagnosis of myelin-associated pathologies in the brain and spinal cord such as multiple sclerosis, as well as stroke and axonopathies [23-26]. We note that we use the same microscope objective with low NA in our system, which may reduce the performance of WM-SORS. However, this paves the way for developing an all-fibre based portable Raman/OCT probe [10,14], which uses comparable NA for both modalities. Using such a compact probe, this combined technique would allow more efficient biological monitoring of traumatic CNS injury over time more so than Raman spectroscopy alone due to the ability to screen tissue at deeper depths guided by OCT [27]. Furthermore, as clinical diagnosis of brain and spinal cord pathologies already rely upon magnetic resonance imaging (MRI), inclusion of WM-SORS/OCT into this platform will add additional noninvasive investigations at depth, allowing for more precise monitoring of disease processes. We acknowledge Raman imaging is still slow compared to OCT imaging. However there is potential for this to be improved by using surface enhanced Raman technologies [28] or even moving to nonlinear approaches, such as coherent anti-Stokes Raman spectroscopy (CARS) [29] or stimulated Raman spectroscopy (SRS) [30].

Acknowledgements. All Authors thank the UK Engineering and Physical Sciences Research Council (EPSRC: EP/J01771X/1, EP/M000869/1), a European Union FAMOS project (FP7 ICT, 317744) and the RS MacDonald Charitable Trust for funding. KD acknowledges support from a Royal Society Leverhulme Trust Senior Fellowship.

Key words: Raman spectroscopy, optical coherence tomography, turbid media, brain tissue.

\section{References}

[1] N. Vogler, S. Heuke, T. W. Bocklitz, M. Schmitt, and J. Popp, Annu. Rev. Anal. Chem. 8(May), 359-87 (2015).

[2] N. Sudheendran, J. Qi, E. D. Young, and A. J. Lazar, Laser Phys. Lett. 105602, 105602 (2014).

[3] C. H. Liu, J. Qi, J. Lu, S. Wang, C. Wu, W. C. Shih, and K. V. Larin, J. Innov. Opt. Health Sci. 08(04), 1550006 (2015).

[4] C. A. Patil, N. Bosschaart, M. D. Keller, T. G. van Leeuwen, and A. Mahadevan-Jansen, Opt. Lett. 33(10), 1135-1137 (2008).

[5] J. W. Evans, R. J. Zawadzki, R. Liu, J. W. Chan, S. M. Lane, and J. S. Werner, J. Biophoton. 2(6-7), 398-406 (2009).

[6] C. A. Patil, J. Kalkman, D. J. Faber, J. S. Nyman, T. G. van Leeuwen, and A. Mahadevan-Jansen, J. Biomed. Opt. 16(1), 011007 (2011).

[7] P. C. Ashok, B. B. Praveen, N. Bellini, A. Riches, K. Dholakia, and C. S. Herrington, Biomed. Opt. Express 4(10), 2179-2186 (2013).

[8] K. M. Khan, H. Krishna, S. K. Majumder, K. D. Rao, and P. K. Gupta, J. Biophotonics 7(1-2), 77-85 (2014).

[9] J. R. Maher, O. Chuchuen, M.H. Henderson, S. Kim, M. T. Rinehart, A. D. M. Kashuba, A. Wax, and D. F. Katz, Biomed. Opt. Express 6(6), 2022 (2015). 
[10] J. Wang, W. Zheng, K. Lin, and Z. Huang, Opt. Lett. 41(13), 3045-3048 (2016).

[11] T. Vankeirsbilck, A. Vercauteren, W. Baeyens, G. Van der Weken, F. Verpoort, G. Vergote, and J. P. Remon, Trends Anal. Chem. 21(12), 869-877 (2002).

[12] K. Buckley and P. Matousek, J. Pharm. Biomed. Anal. 55(4), 645-652 (2011).

[13] P. R. T. Jess, M. Mazilu, K. Dholakia, A. C. Riches, and C. S. Herrington, Int. J. Cancer 124(2), 376-380 (2009).

[14] C. A. Patil, H. Kirshnamoorthi, D.L. Ellis, T. G. Van Leeuwen, and A. Mahadevan-Jansen, Lasers Surg. Med. 43(2), 143-151 (2011).

[15] J. Schleusener, C. Reble, M. C. Meinke, and J. Helfmann, Photonics and Lasers in Medicine 4(2), 151-167 (2015).

[16] P. Matousek, M.D. Morris, N. Everall, I. P. Clark, M. Towrie, E. Draper, A. Goodship, and A. W. Parker, Appl. Spectrosc. 59(12), 1485-1492 (2005).

[17] J. R. Maher and A. J. Berger, Appl. Spectrosc. 64(1), 61-65 (2010).

[18] M. D. Keller, S. K. Majumder, and A. Mahadevan-jansen, Opt. Lett. 34(7), 926-928 (2009).

[19] M. D. Keller, E. Vargis, N. de Matos Granja, R. H. Wilson, M. Mycek, M. C. Kelley, and A. Mahadevan-Jansen, J. Biomed. Opt. 16(7), 077006 (2011).

[20] Z. Liao, F. Sinjab, G. Gibson, M. Padgett, and I. Notingher, Opt. Express 24(12), 12701 (2016).

[21] A. C. De Luca, M. Mazilu, A. Riches, C. S. Herrington, and K. Dholakia, Anal. Chem. 82(2), 738-745 (2010).

[22] M. Chen, N. McReynolds, E. C. Campbell, M. Mazilu, J. Barbosa, K. Dholakia, and S. J. Powis, PLOS ONE 10(5), e0125158 (2015).

[23] A. P.D. Henderson, S. A. Trip, P. G. Schlottmann, D. R. Altmann, D. F. Garway-Heath, G. T. Plant, and D. H. Miller, Brain 131(Pt 1), 277-87 (2008).

[24] Y. Shi, D. Zhang, T. B. Huff, X. Wang, R. Shi, X. M. Xu, and J. X. Cheng, J. Biomed. Opt. 16(10), 106012 (2011).

[25] G. C. Munguba, S. Galeb, Y. Liu, D. C. Landy, D. Lam, A. Camp, S. Samad, M. L. Tapia, and R. K. Lee, Invest. Ophthalmol. Vis. Sci. 55(10), 6505-6513 (2014).

[26] S. Chen, Q. Liu, X. Shu, B. Soetikno, S. Tong, and H. F. Zhang, Biomed. Opt. Express 7(9), 3377 (2016).

[27] J. M. Surmacki, L. Ansel-Bollepalli, F. Pischiutta, E. R. Zanier, A. Ercole, and S.E. Bohndiek, Analyst 142(1), 132-139 (2017).

[28] N. Stone, M. Kerssens, G. R. Lloyd, K. Faulds, D. Graham, and P. Matousek, Chem. Sci. 2(4), 776 (2011).

[29] C. L. Evans, E. O. Potma, M. Puoris'haag, D. Côté, C.P. Lin, and X. S. Xie, Proc. Natl. Acad. Sci. USA 102(46), 16807-16812 (2005).

[30] B. Saar, C. Freudiger, J. Reichman, M. Stanley, G. Holtom, and X. Xie, Science 330(December), 1369-1370 (2010).

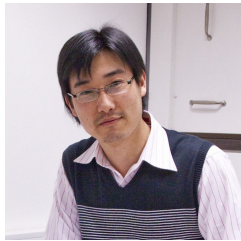

Mingzhou Chen is a postdoctoral research fellow at University of St. Andrews. He completed his PhD at University of Pretoria (South Africa) in 2009. His main research interests are Raman spectroscopy, OCT, optical trapping, singular optics, laser beam correction and complex optical wave fields.

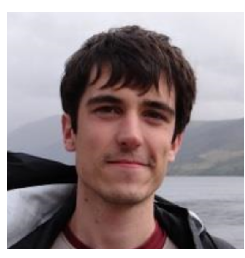

Josep Mas studied Physics at the University de Barcelona (Catalunya, Spain) where he specialized in Optics and Astronomy (2003-2007), and he later obtained a MSc in Photonics from UPC, UB, UAB, and ICFO (Barcelona, 2008). He joined the Optical Trapping Lab Grup de Biofotnica at the University of Barcelona for carrying out his $\mathrm{PhD}$ studies in the field of holographic optical tweezers and force sensing in living cells, under the supervision of Estela Martn-Badosa and Mario Montes-Usategui. He also spent part of his PhD at the Niels Bohr Institute (Copenhagen) working on optical trap calibration in viscoelastic biological media.

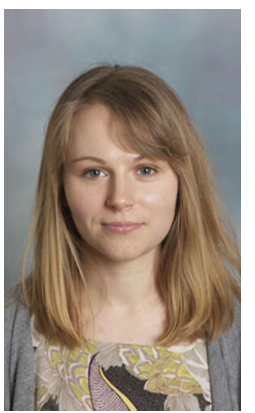

\section{Lindsey H. Forbes .}

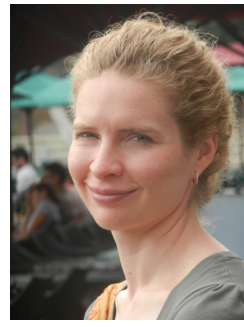

Melissa R. Andrews received her B.Sc. in Bioengineering from the University of Illinois at Urbana-Champaign in 2000 and $\mathrm{PhD}$ in Anatomy and Cell Biology from the State University of New York Upstate Medical University in 2006. Her post-doctoral work was done at the Cambridge Centre for Brain Repair, followed by a Lectureship in Anatomy at the University of St Andrews School of Medicine. She is currently a Lecturer in Systems Physiology at the University of Southampton. Her current research centre on investigating novel mechanisms of promoting brain and spinal cord repair following traumatic injury using cellular transplantation and gene therapy. 


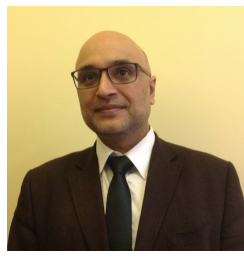

Kishan Dholakia is Professor of Physics at the University of St Andrews Scotland and an honorary adjunct Professor at the Centre for Optical Sciences at the University of Arizona, USA, visiting Professor at Chiba University, Japan and distinguished Professor at IIT Madras, India. He heads the Optical Manipulation Group who work on a wide range of topics based on shaped light. This includes advanced imaging for neuroscience and cancer diagnosis and optical manipulation. His work has led to a citation in the Guinness Book of World Records. He is a Fellow of the Royal Society of Edinburgh, the Optical Society (OSA) and SPIE. He won the European Optics Prize in 2003 and in 2008 he received a Royal Society Wolfson Merit Award. He is the 2016 recipient of the R.W. Wood Prize of the Optical Society and the 2017 recipient of the Thomas Young Medal and Prize of the Institute of Physics. 